\title{
Con la microscopia a scansione di forza alla scoperta delle organizzazioni molecolari in biologia*
}

\author{
B. SAMORI $* *$
}

\begin{abstract}
A Microscopia de Força Atómica (Atomic Force Microscopy, AFM) ou Microscopia de Varrimento de Força (Scanning Force Microscopy, SFM) é seguramente a mais recente novidade no campo microscópico. Nascida nos laboratórios da IBM (nos quais nasceu também o Laser) em 1986, ao contrário das outras técnicas microscópicas de nível molecular baseadas sobre interacções de fotões ou electrões $\mathbf{e}$ a matéria ela baseia numa ideia ao mesmo tempo simples e original:

cio si basa sull' utilizzo di una sonda che scorre sul campione in esame ed effettua su di esso misure di una proprieta locale. Una sonda come puo esserlo uno stetoscopio che registra localmente dei suoni.

Su questo nuovo approccio sono basate le microscopie a scansione di sonde (scanning probe microsocpies). Le immagini nascono dalla composizione di dati misurati localmente, punto per punto separatamente e la risoluzione dipende da quanto "Iocale" sia stata la misura.
\end{abstract} 'tocar' com uma agulha de dimensões atómicas (raio de curvatura da ponta de $30 \mathrm{~nm}$ ) os campos de força moleculares (geralmente forças de Van der Waals) que formam a superfície de uma molécula (como a vara com a qual um cego sente a organização superficial do espaço em volta dele). Embora a sua utilização abranja a física, a química e a biologia, foi no campo da biologia molecular e celular que a AFM conseguiu os seus maiores sucessos e isso pelo facto de ser a resolução da SFM, hoje em dia, apenas capaz de 'ver' conjuntos macromoleculares como a cromatina ou complexos membrano-proteicos. Com efeito com essa técnica conseguiu-se determinar a quiralidade da cromatina e a organização de complexos membrano-proteicos em solução e esse último facto é a outra vantagem da SFM: analisar de maneira não invasiva (e no seu ambiente) as amostras biológicas.

E' nato negli anni 80 un nuovo approccio alla microscopia con potenzialità applicative alla biologia strutturale che si vanno scoprendo essere sempre più sorprendenti. In questo nuovo modo di fare microscopia non vi sono più sonde di fotoni od elettroni che colpiscono il campione. Non vi sono più problemi di risoluzioni laterali limitate dalle lunghezze d'onda di queste radiazioni impiegate. Non più lenti con le loro aberrazioni. Non più diaframmi. II nuovo approc-
La microscopia a sonda che più si sta rivelando utile in biologia è la Atomic Force Microscopy $(1,2)$. Questa denominazione viene sempre più diffusamente sostituita con Scanning Force Microscopy (SFM), dizione più corretta dal punto di vista fisico, che tradurremo in Microscopia a scansione di forza.

\section{COME E NATA?}

Agli inizi degli anni 80, nei laboratori IBM a Ruschlikon (Svizzera) Heini Rohrer e Gerard Binning srilupparono la Scanning Tunneling Microscopy e per questa invenzione nel 1986 fu loro assegnato il premio Nobel in Fisica. Questa nuova microscopia era basata sull' effetto tunnel. Quando due conduttori metallici (in questo contesto, uno dei due è uno stilo appuntito) sono posti a distanze dell'ordine dell' $\AA$ e separati da uno strato non conduttore (vuoto 0 aria), una piccolissima corrente elettrica fluisce dall' atomo più avanzato della punta dello stilo verso l'atomo a lui più vicino della superficie dell' altro conduttore.

La genesi di questo fenomeno deriva dal comportarsi degli elettroni sia come particelle sia come onde. Gli elettroni si muovono liberamente all' interno dei materiali conduttori e vengono riflessi indietro ove questi materiali finiscono. Ma questi elettroni non si comportano come le palle da tennis quando esse rimbalzano su un piano limite, ad esempio su una parete: la riflessione degli elettroni può non accadere sempre esattamente sullo stesso piano limite. Una parte di loro infatti penetrerà anche in quella Sottilissima lamina di vuoto che si trova fra i due conduttori. Se poi fra questi viene applicata una tensione elettrica, essa stabilisce un flusso di corrente che possiamo poi modulare a piacere, al variare della tensione stessa e della distanza della punta dalla superficie Questa corrente di tunneling è estremamente localizzata. Rohrer, Binning e Gerber pensarono quindi di pilotare la punta facendola scorrere da un atomo a quelli adiacenti sulla superficie in esame sì da evidenziare la loro presenza e posizione. Utilizzarono a tale scopo la tecnologia dei piezoelettrici che consente spostamenti dello stilo in tutte le direzioni spaziali con precisioni dell' ordine dei decimi di Å, quindi all' interno di dimensioni subatomiche. Le correnti di tunneling vengono così registrate punto per punto, Å per $\AA$, sulla superficie e posti insieme, come pezzi di un puzzle, si da ottenere una topografia della superficie esaminata. Con questa tecnica riuscirono a raggiungere risoluzioni laterali inferiori alla lunghezza d' onda degli elettroni delle correnti di tunnel che misuravano. Anche nel caso dello stetoscopio che abbiamo preso come modello macroscopico delle sonde, I' area su cui effettua la misura è inferiore alla lunghezza d'onda del suono che registra.

In questi ultimi anni sono state tentate applicazioni di questa microscopia alla biologia. Tali esperimenti hanno confermato la diffusa sensazione che questa tecnica non possa essere di grande utilità in questo contesto perche richiede conduzioni elettroniche che $\mathrm{i}$ materiali organici e biologici, di norma isolanti, non sono in grado di assicurare.

Lo stesso Binning nel 1986, già conscio di queste limitazioni intrinseche all' STM, in collaborazione con Carl Quate di Standford, estese questo nuovo approccio della microscopia a scansione di sonde a misure che non richiedessero fenomeni di conduzione.

Due atomi che stanno l'uno di fronte all' altro possono awertire la loro 
presenza anche attraverso le forze attrattive 0 repulsive che essi possono esercitare I' uno sull' altro. Essi mostrarono come fosse possibile misurare queste interazioni fra gli atomi su una punta in scansione e quelli sulla superficie del campione in esame. Nacque così quella che essi chiamarono la Atomic Force Microscopy. Al contrario della microscopia ad efietto tunnel, con questa microscopia, non essendo più in gioco alcuna conduzione elettronica, possiamo anche esaminare materiali isolanti ed in particolare campioni biologici.

Di fatto questo microscopio non è altro che un profilometro in scansione che può operare fino alla scala molecolare. E' sorprendente vedere come esso non sia nato da una evoluzione dei profilometri macroscopici usati fin dall' inizio del secolo nello studio della rugosita di superfici levigate meccanicamente. Sarebbe bastato lavorare un poco di più sull'affinamento della sonde allora usate per giungere direttamente a tale tecnica Essa è nata invece come uno sviluppo della microscopia ad effetto tunnel, una tecnica concettualmente molto più raffinata e più complessa. E' questo un altro esempio di come il progresso scelga a volte cammini ben più tortuosi $\mathrm{e}$ ad energie potenziali ben superiori a quelle di altri che avrebbero potuto condurre alle stesse acquisizioni.

\section{COME FUNZIONA?}

Le risoluzioni di questo nuovo profilometro potranno diventare anche molecolari se la sonda utilizzata sarà sottile grossomodo come la molecola che si desidera osservare e se si riuscirà a far scorrere questa sonda su di essa, toccandola con una delicatezza sufficiente da non modificarne la struttura Registrando punto per punto la posizione in altezza dello stilo si potrà così ottenere una mappa tridimensonale della molecola in esame. Si trasferiscono così le nostre esperienze tattili alle dimensioni del mondo delle singole molecole ed alla Ioro delicatezza.

Negli strumenti che lavorano in scansione continua sul campione (contact-mode) (figura 1) questo è stato reso possibile posizionando la punta su una leva, di norma triangolare, molto flessibile, con costanti elastiche da 0.1 a $0.01 \mathrm{~N} / \mathrm{m}$. La costante elastica di una striscia di Al lunga $4 \mathrm{~mm}$ e larga $1 \mathrm{~mm}$ è di circa $1 \mathrm{~N} / \mathrm{m}$.

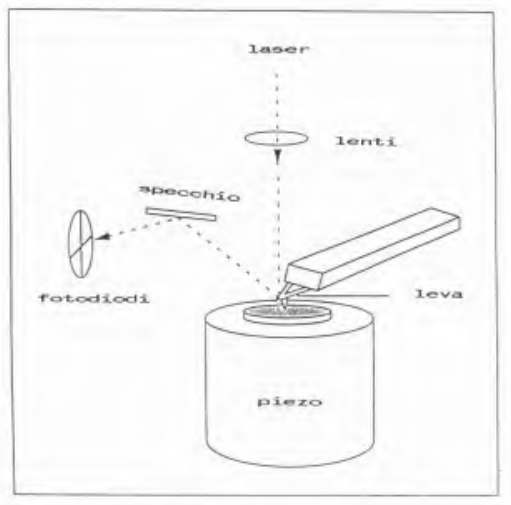

1. Schema costruttivo di un microscopio a scansione di forza. La sonda è sostenuta da una leva a sezione triangolare. I suoi spostamenti In verticale indotti durante la scansione dalla rugosità del campione sono amplificati e misurati con una leva ottica; un raggio laser si ritlette sulla estremita del braccio che supporta la punta e va a colpire una serle di fotodiodi afflancati. Lo scorrimento del campione e la definizione della sua posizione sono affidati ad un piezoelettrico che ne controlla i movimenti con una precisione superiore al decimo di $\AA$.

La leva deve essere così flessibile perchè deve assorbire gli spostamenti della punta durante la scansione si da evitare ogni danno al materiale in esame. Saranno in gioco costanti elastiche dell' ordine di $10 \mathrm{~N} / \mathrm{m}$ se pensiamo ad un legame chimico fra due atomi. Saranno in gioco costanti ben minori se vogliamo preservare quegli ordini supramolecolari che in biologia giocano ruoli funzionali di primaria importanza.

Gli spostamenti in verticale della punta sono amplificati e misurati con una leva ottica: un raggio laser si riflette sulla estremità del braccio che supporta la punta e va a colpire una serie di fotodiodi affiancati. Lo scorrimento del campione e la definizione della sua posizione sono afiidati ad un piezoelettrico che ne controlla i movimenti con una precisione superiore al decimo di Å.

In biologia, per meglio controllare i possibili danni della sonda all' integrita del campione, si opera normalmente misurando e tenendo costante in ogni punto la forza esercitata dalla sonda sul campione: quando la sonda incontra un ostacolo la leva si piega ed un circuito elettronico di controllo allontana il campione fino a completa rimozione della deflessione stessa. L' immagine e cosi ottenuta registrando punto per punto, I' altezza del campione richiesta per mantenere la forza costante.

E' stata di recente sviluppata una nuova tecnica di scansione detta "tapping-mode" (figura 2). La leva è più rigi- da, con una costante elastica di circa 50 $\mathrm{N} / \mathrm{m}$. Mentre il campione viene posto in scansione laterale la leva è posta in vribrazione nell' intorno della sua frequenza di risonanza $(250-300 \mathrm{kHz})$ con ampiezze, al momento, ancora dell' ordine dei 10-20 $\mathrm{nm}$. II campione aggancia e riduce I' ampiezza dell' oscillazione. In questo caso è la riduzione dell' ampiezza ad essere tenuta costante dal circuito di controllo e la topografia del campione è ottenuta registrando gli spostamenti del piezo richiesti per mantenere questa condizione. II tapping-mode ha almeno due vantaggi sul metodo a contatto. Primo, le forze punta-campione sono minori. Secondo, le forze tangenziall di scorrimento sono ridotte al minimo: la forza esercitata è quasi completamente perpendicolare alla superficie del campione e sono così su di esso minimizzati gli effetti distorsivi della punta. II tapping-mode che si riteneva fosse limitato ad operare in arla, è stato di recente esteso anche all' ambiente fisiologico dal gruppo di Paul e Helen Hansma (3). Con questo nuovo sviluppo essi hanno mostrato come si possa segulre in soluzione non solo il moto di singole molecole di DNA debolmente attaccate al substrato di mlca, ma anche la loro degradazione ad opera della DNasI I (3).

2. Nel contact-mode (a) la punta scorre sul campione senza perdere in contatto con esso. Nel Tapping-mode essa salta invece da un punto a quello contiguo (da referenza 3).
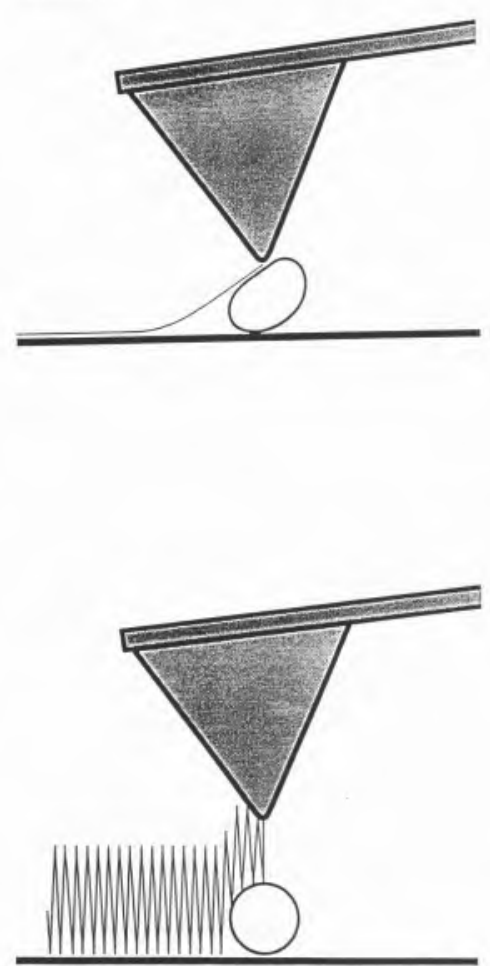


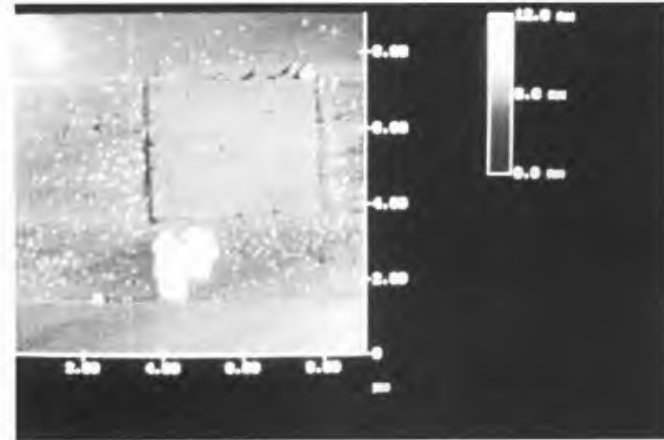

3. L' osservazione di materiali biologici soffici e non legati saldamente al substrato può portare, se non si riesce a minimizzare le interazioni punta-campione, alla sua distruzione e ad artefatti simili a queili mostrati da questa figura Allargando il campo di osservazione posso vedere aree quadrate corrispondenti alla scansione precedente in cui la sonda invece di fare immagini ha "arato" la superficie del campione spostando le molecole di DNA depositate sulla superficie della mica al bordi della zona di scansione

\section{LE FORZE IN GIOCO}

II collaudo di uno strumento commerciale prevede di norma, l'osservazione degli atomi della superficie di un cristallo di mica. Ma se si vuole passare dai cristalli a materiali ben più soffici e delicati come i materiali biologici i glochi diventano del tutto diversi. Se si vogliono osservare molecole depositate su un substrato come la mica, il risultato che più facilmente posso ottenere è quello di arare il mio campione con la punta è spostare il materiale In esame al bordi del campo di osservazione (figura 3). Se si vogliono osservare invece tessuti 0 complessi molecolari, la sonda può deformarli facilmente e le proprietà viscoelastiche del campione giocano un ruolo primario nella formazione delle Immagini.

Quando lo stilo si avvicina al campione entrano in gioco forze sia a corto sia a lungo raggio, che operano su aree della punta più o meno ristrette.

Le forze a lungo raggio sono di norma attrattive e composte da forze di van der Waals, forze elettrostatiche e caplllari $(4,5)$. Le prime dipendono dalla forma e dal raggio di curvatura finale della punta Queste forze sono dell' ordine di $10^{-9} \mathrm{~N}$. Le forze elettrostatiche nascono dalle cariche superficiali presenti e possono essere molto più forti, fino a $10^{-7} \mathrm{~N}$. II campione è sempre ricoperto da uno strato di acqua se si opera in aria. Quando la punta si avvicina si forma qulndi un menisco che la tiene aggancia- ta al campione stesso. Queste forze capillari possono essere anch' esse dell' ordine di $10^{-7} \mathrm{~N}$.

In un sistema macroscopico queste grandezze sono Infinitesime, ma nelle scale su cui opera questa tecnica si possono con esse sviluppare pressioni locall enormi: l' area di contatto punta-campione è estremamente piccola. E' immediato vedere come con forze di soli $10^{-10} \mathrm{~N}$ e con aree di contatto di circa $200 \AA^{2}$, quali quelle attese nel caso delle punte commerciali piramidali, si sviluppino localmente pressioni dell' ordine delle 10 atmosfere. Queste pressioni non possono non essere fonte di grande preoccupazione se si vogliono studiare campioni biologici.

Le forze a corto raggio sono repulsive e sono dovute alla pressione meccanica che la punta esercita sul campione. Esse possono creare profonde deformazIoni locall In un campione particolarmente soffice.

Queste forze devono essere ridotte al massimo possibile. Per fare questo si deve primariamente agire sullo stilo ridurcendone la dimensione e controllandone la composizione chimica, anche in funzlone di quella del campione. Si deve inoltre eliminare le forze capillari operando a umidità controllata (almeno sotto il $30 \%)$ o sotto liquidi. Si devono usare modalità di scansione della punta II meno Invasive possibili: il tapping mode è di norma preferibile al contact mode. Se si utilizza quest' ultimo si deve, prima di iniziare la scansione, ritrarre la punta, tenuta incollata al campione dalle forze attrattive, fino quasi al punto di distacco e operare quindi la scansione continuando a ritrarre la punta.

\section{IMMAGINI, E NON SOLO, DI TESSUTI E CELLULE IN AMBIENTE FISIOLOGICO}

II problema della deformazione del campione ha spinto alcunl biologi a partire nel loro studi con un microscopio SFM da materiali ben duri come i denti. Ottennero così facilmente immagini delle strutture cristalline dello smalto e del legamenti paradontali di collagene. I risultati ottenuti non fornirono di fatto alcun avanzamento sul piano conoscitivo rispetto a quanto era stato In precedenza ottenuto con la microscopla elettronica. Quelle ImmagInl SFM erano state però reglstrate su denti tenuti immersi in una soluzione tampone. Gli autori pensarono di aggiungere a questa un poco di acldo ortofosforico e filmare il suo attacco acldo sullo smalto registrando Immagini In tempi successivi (6). Condussero I' esperimento anche con Coca Cola, ma al momento non hanno ancora deciso di pubblicizzare i dati. Questo è un esemplo di come tale tecnica ci permetta non solo di poter osservare campioni biologici con risoluzione anche molecolare ed in ambiente di fatto fisilogico, ma anche di filmare processi che avvengono in essi con tempi di scala almeno dell' ordine di qualche secondo. E' questo il tempo minimo richiesto per ottenere un' immagine. Ciò è stato mostrato in film costruiti registrando in sequenza continua immagini dei processi di coagulazione del sangue (7) 0 dell' esocitosi di virus da cellule (8) e piu recentemente dal gia menzionato esperimento di degradazione di molecole di DNA ad opera della DNasi I (3).

Nel caso di cellule viventi nei loro mezzl di coltura si può non solo osservarle e seguire processi che avvengono sulla Ioro superficie come la propagazione di ondulazioni (9), ma anche fermare lo stilo In un punto ed effettuare misurazioni locail di elasticita della stessa superficie (10). Questa prospettiva di effettuare misure locall sta aprendo possibilità di utilizzo di queste tecnica in biologia che potranno essere particolarmente utili e significative. Sono in corso su questa linea esperimenti particolarmente eleganti come seguire la risposta dinamica di cellule cardiache a diverse concentrazioni di ioni calcio nel mezzo di coltura ed in presenza 0 meno di farmaci complessanti (P. Hansma, comunicazione personale). $E^{\prime}$ in corso di effettuazione anche uno studio dei processi di cicatrizzazione e riparazione in cornee di coniglio: ne registriamo immagini e ne misuriamo localmente la elasticità a tempi crescenti da un intervento chirurgico (11).

\section{LE SONDE: LA LORO FORMA, LA LORO COMPOSIZIONE}

Questi risultati fino ad ora menzionati sono stati ottenuti su una scala ancora macroscopica e sono ben Iontani dalla risoluzione molecolare. Le risoluzioni sono in biologia primarlamente limitate dalla deformabilità del campione $\mathrm{e}$ dalla dimensione delle punte.

Le punte commerciali sono prodotte con tecniche di microfabbricazione 


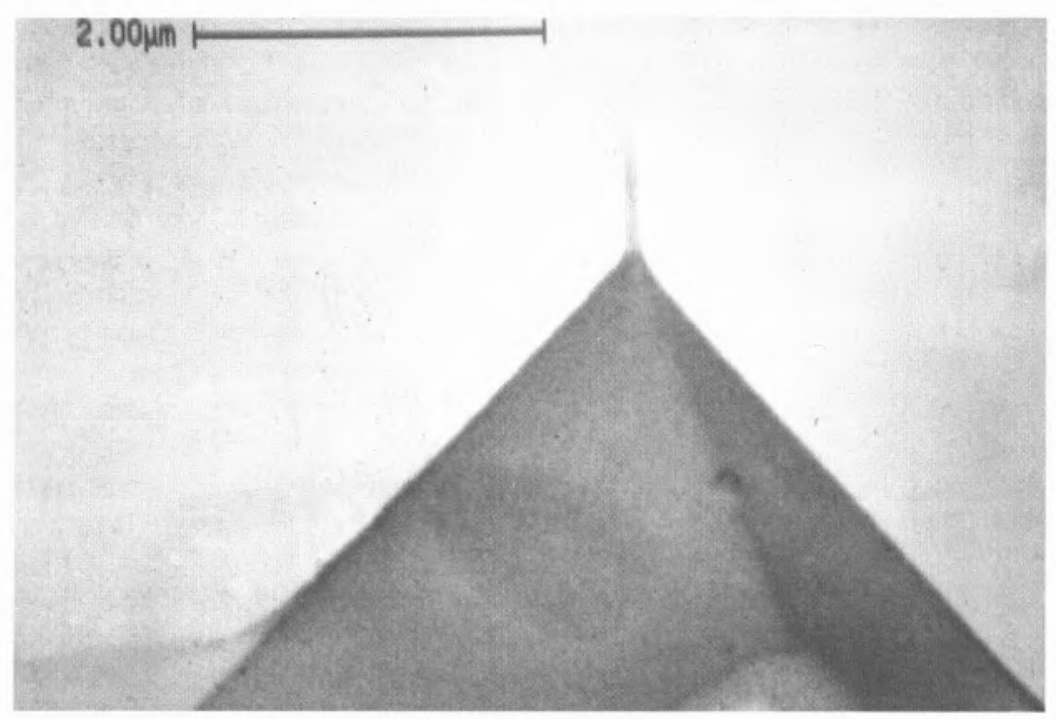

A

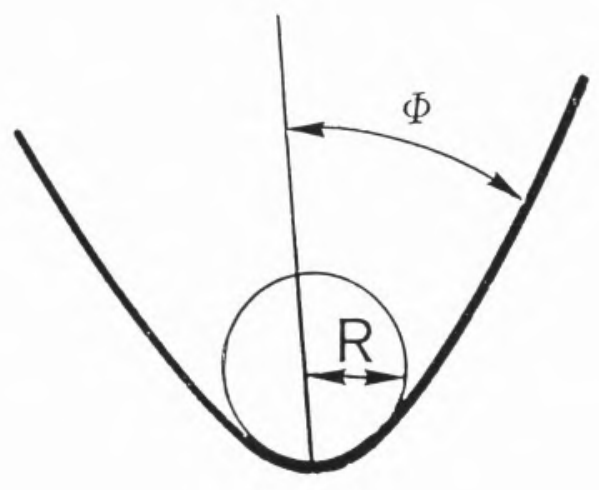

B
4. (A) Immagine con un microscopio elettronico a scansione di una delle super-tips prodotte da noi nell Università della Calabria (Cosenza). Sulle punte commerciali a profilo piramidale, usate nelle grande maggioranza del laboratori operanti nel settore, si fanno crescere, con la tecnica di D. Keller (12), aghi di carbonio amorfo con raggi di curvatura terminali inferiori anche di un ordine di grandezza (vedi testo). (B) La forma delle punte determina la loro capacita risolutiva. La risoluzione orizzontale dipende dai raggio terminale di apertura ( $R$ ) e quella verticale dall' angolo di apertura della punta.

sviluppate nell' Industria elettronica. I loro raggi di curvatura finall sono mediamente dell' ordine dei $30 \mathrm{~nm}$ e l' angolo di apertura sui $57^{\circ}$ (figura 4). so questa proprietà è assolutamente richiesta. Ne consegue che per operare al meglio In biologia con questa nuova microscopia bisogna poterle affiancare, anche solo con un ruolo anciliare, un microscopio elettronico, possibllmente a scansione e ad emissione di campo per la più ridotta larghezza del suo fascio elettronico.

Con queste punte nel laboratori di Carlos Bustamante a Eugene(OR) ed in quello di Helen Hansma a S. Barbara (CA) si stanno al momento studiando complessi nucleoproteici ed i processi di docking che portano alla loro formazione. Sono state di recente riportate dal prlmo gruppo immagini del complesso fra l' RNA polimerasi e frammenti di DNA contenenti l' opportuno promotore.

Queste immagini registrate in arla con le punte di carbonio sono state in grado di dimostrare chiaramente come II bending del DNA sul sito di legame della polimerasi cambi quando si passi dal complesso iniziale a quello di elongazione con un trascritto di appena quindici nucleotidi (13).

\section{LA CHIRALITÀ DEL SUPERAVVOLGIMENTO DEL DNA}

Le immagini costruite da questa microscopia essendo di fatto del profill topografici, contengono informazioni sulla tridimensionalità dell' oggetto In esame. Le immagini registrate con un microscopio elettronico nascono da una sovrapposizione bidimensionale dei diversi strati della struttura tridimensionale studiata. Si possono prendere coppie stereo e immagini ad angoli diversi. Con i microscopi a trasmissione (TEM) è difficile ottenere informazioni sulla struttura superficiale e la natura tridimensionale dell' oggetto in esame. Su questo si basa la forte complementarietà fra la microscopia elettronica a trasmissione e quelle a scansione di forza.

In uno studio comparativo fra le microscople TEM e SFM su complessi sinaptonemici è ben mostrato come le immagini TEM non siano di rivelare dettagli strutturali resi invece perfettamente evidenti da quelle SFM (14).

Quando si scende a risoluzioni molecolari anche nelle immagini SFM la tridimensionalita dell' oggetto è difflcilmente riconoscibile direttamente. Con una buona punta di carbonio si possono ora agevolmente ottenere immagini assolutamente necessarie, non tanto perchè queste assicurano forti rlduzioni nel raggio di curvatura (flgura 4), quanto perchè esse sono molto più Idrofobiche delle sonde commerciali a base di sillcio e se si vuole operare In amblente acquo- 


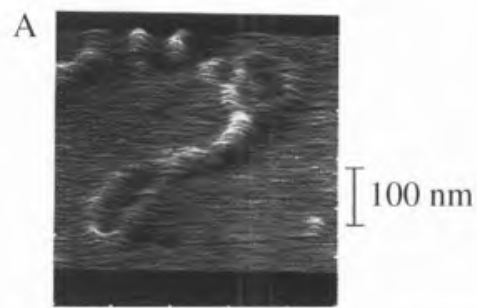

B
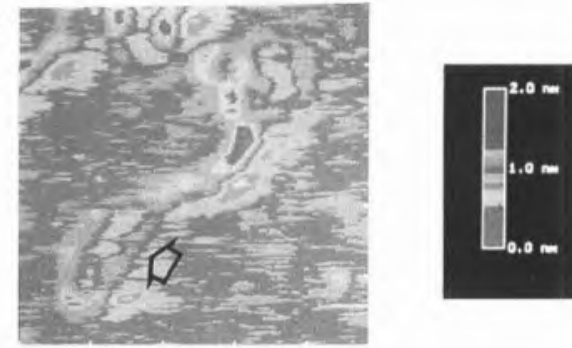

C

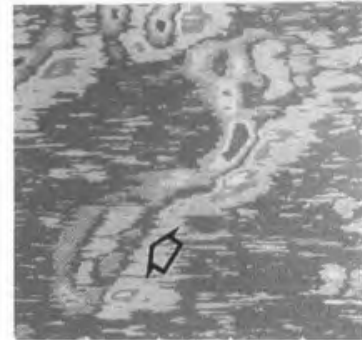

5.

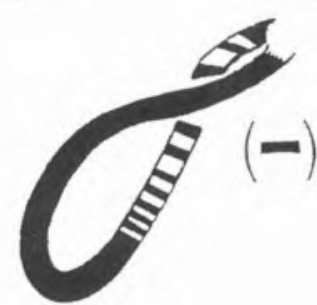

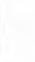

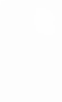

\section{5. (A) Immagine di una singola molecola di DNA circolare.}

Nonostante $l$ alta definizione di questa immagine non si è però in grado di assegnare direttamente la chiralita del superavvolgimento della molecola di plasmide in essa "fotografato". Non si è in grado di identificare agli incroci delle catene quale di esse sia sopra e quale sia sotto. E' peró possibile trattare l' immagine si da colorare con otto colori diversi otto sezioni della stessa che sono ad altezze diverse sull'asse verticale. Prima si posiziona il colore rosa si da avere tutta la spira che si vuole esaminare compleatmente colorata con esso (8). Spostando in alto questo colore un braccio della spira Inizia ad interrompersi nei punti indicati dalle frecce. Quindi questo braccio è sotto l' altro che resta invece continuo. Si puo cosi facilmente schematizzare la spira come indicato ed identificare la chiralità come destrogira e quindi il superavvolgimento come negativo (da reterenza 15).

quale quella riportata in figura $5 \mathrm{~A}$. Nonostante la sua definizione particolarmente buona, la sua risoluzione verticale non permette però di riconoscere agli incroci quale braccio della catena sia sopra e quale sia sotto: non si riesce cioè ad assegnare la chiralità del superavvoigimento. Per rivelare le informazioni tridimensionali di queste immagini abblamo messo a punto una tecnica di indagine stratigrafica delle stesse (15). Sono ampiamente diffusi programmi di elaborazioni di immagini, quall I' NIH image (Bethesda, MD) 0 il TCL-Image (Technical University of Delft). Nel Microscopio SFM Nanoscope III della Digital Inst. (S.
Barbara, CA), è già disponibile un programma che al pari di quelli permette di evidenziare con diversi colori sezioni delle immagini che sono ad altezze diverse sull' asse verticale. In questo caso si può avere la spira che si vuole esaminare colorata di un unico colore. Nella figura $5 \mathrm{~B}$ questo è stato scelto essere il rosa.

Questo colore può essere quindi spostato in alto 0 In basso sull' asse verticale sino ad identificare agll incroci delle catene quale di esse sia sopra all' altra. Spostando in alto il colore che nella figura 5B si estendeva su tutta la spira, un braccio di questa inizia ad interrompersi. Ciò mostra che nei pressi dell' incrocio questo braccio è sotto l' altro che resta invece continuo. Si può così facilmente identificare la chiralità destrogira, cosidetta negativa, del superavvolgimento, come schematizzato nel fondo della figura 5 .

Abbiamo di recente mostrato come con una punta la cui forma presenti raggi di apertura molto piccoli (figura 4) si possa ottenere questa informazione anche senza alcuna analisi stratigrafica, come mostrato dalla figura 6 . La sua risoluzione verticale è talmente buona che i'intreccio del due bracci di catena del DNA sia in questo caso direttamente e chiaramente identificabile nella sua tridimensionallta (16). Mentre la risoluzio- ne laterale dipende dal raggio di curvatura terminale della punta, la risoluzione verticale dipende invece dal suo raggio di apertura.

\section{ANALISI TOPOLOGICA DI SINGOLE MOLECOLE}

Le nostre conoscenze sulle molecole sono state fino ad ora sempre mediate su loro popolazioni grandissime contenenti un numero di molecole la cui unita di misura è il Numero di Avogadro di esse (6.02 10 $10^{-23}$ molecole). Questo offre il vantaggio di avere a che fare con grandezze stazionarie che possono essere interpretate in termini termodinamici. Ma così si e sviluppata una conoscenza di parametri strutturali e costanti dinamiche che sono di fatto limitate in quanto mediate su distribuzioni molecolari non note e non necessariamente omogenee. Quanto più aumenta la complessità delle molecole, dei sistemi studiati e delle loro funzioni tanto più si fa importante avere informazioni sulle proprietà delle singole molecole e possibilmente anche sulle funzioni di distribuzione di queste loro proprietà

Immagini come quella in figura $6 \mathrm{ci}$ permettono di estendere I' analisi topologica del superavvolgimento di un campione di DNA. II superavvolgimento è di norma descritto da un parametro topologico come il linking number" $\left(L_{k}\right)$. Questo viene misurato con la gel elettroforesi. Per le larghe popolazioni di molecole costituenti un campione devo usare un parametro puramente topologico, come l' $L_{k}$, che è invariante alla conformazione delle singole molecole. Questo parametro mi può così fornire una valutazione topologica delle concatenazioni delle catene nelle molecole del mio campione. Questa valutazione è necessariamente mediata non solo su tutte queste le molecole ma anche su tutte le conformazioni che esse assumono nel tempo richiesto per la loro separazione elettroforetica in un' unica banda.

La possibilità di analizzare una singola molecola alla volta permette di ottenere una descrizione ben più completa. Posso così utilizzare un vero prametro geometrico come il writing $\left(\mathrm{W}_{\mathrm{r}}\right)$. Per ottenerio, proprio perchè è un parametro geometrico, devo conoscere la struttura nelle tre dimensioni del superavvolgimento della doppia elica. Immagini come quella in figura 6 ci rendono possibile calcolare $\| W_{\text {, della molecola }}$ 
fotografata. questo parametro può essere stimato o calcolato esattamente come I' integrale di Gauss della curva descritta dalla catena Per la molecola di figura 6 il $W_{r}$ può essere stimato essere molto vicino a valore di -2 , col primo metodo, ed è pol stato da noi calcolato, con il secondo, essere esattamente 1.975 (17). Su questa base possiamo giungere ad una definizione sperimentale di tutti i parametri utllizzati in topologia come fino ad ora non era considerato essere possibile. Infatti se si parte da un campione contenente solo singoli topoisomeri di DNA superavvolto isolati elettroforeticamente, la loro differenza nell' $L_{k}$ rispetto alla forma rilassata corrispondente (linking deficit) risulta nota. Determinando quindi il $W_{\mathrm{r}}$ di singole molecole dello stesso campione si ottiene così anche il loro twisting $\left(T_{w}\right)$ e la Ioro topologia risulta essere completamente determinata

\section{LE FIBRE DELLA CROMATINA IN TRE DIMENSIONI (18)}

In questi ultimi due decenni la struttura delle flbre di cromatina a bassa concentrazione salina è stata oggetto di una controversia molto vivace. Sono questi stati a più basso impacchettamento nucleosomico quelli che $\mathrm{ci}$ danno più informazioni sulle strutture incontrate dalle polimerasi. Preparazioni con la tecnica di Miller per la microscopia elettronica, già dai primi lavori classici di Thoma, Koller e Klug (19), hanno mostrato, a bassa forza ionica, strutture estese di "perle sul fllo". A forza ionica un poco più alta $(10 \mathrm{mM} \mathrm{NaCl})$ hanno evIdenziato strutture bidimensionali a "zig-zag". Ad ulteriori agglunte di $\mathrm{NaCl}$ fino a $100 \mathrm{mM}$, sembrava che la fibra si compattasse progressivamente per formare la cosidetta struttura a "30 nm". Tuttavia sospetti sulla significatività di queste osservazioni furono gia sollevati dagli stessi autori perchè non si possono escludere distorsioni delle strutture originarie dovute a stiramenti e tensioni durante il processo di essicamento completo ed alle condizioni di alto vuoto richieste dalla microscopia elettronica. Dati di raggi $X$ e scattering neutronico a basso angolo suggerirono prima che i nucleosomi fossero impaccati densamente su una fibra con un diametro di circa $10 \mathrm{~nm}$ (20), poi con una struttura ad elica aperta (21). Quest' ultimo dato fu rafforzato poi da dati di microscopia STEM e da crio-microscopia elettronica (23). La microscopia SFM con I' utilizzo del tapping mode ha iniziato a rivelare già a bassa forza ionica strutture tridimensionali con diametri di circa $34 \mathrm{~nm}$ senza ordini elicoidali. La tecnica di indagine stratigrafica, messa a punto in precedenza per studiare la chiralità del superavvoigimento plectonemico (15) $\mathrm{cl}$ ha permesso di studiare queste strutture evidenziando l' altezza sull' asse verticale del singoll nucleosomi. Sulla base di queste imagini stratigraflche le strutture della fibra di cromatina a bassa forza ionica sono risultate essere ben piu organizzate di quanto accettato fino ad ora ma anche piu irregolari. Questa irregolarita sembra essere imposta dalle costrizioni legate alla rigidità dei tratti di DNA internucleosomiali (linkers) agli angoli di entrata ed uscita del filamento del DNA sul nucleosoma ed alla presenza del linker istonici. Aumentando la forza ionica aumenta la flessibilltà del linkers ma anche I' interazione fra nucleosomi contigui: si possono cosi ottenere le strutture più ordinate che questa tecnica ha mostrato a partire da soluzioni $10 \mathrm{mM}$. Questo studio ancora in corso sta mostrando come la struttura della cromatina risulti da un compromesso fra le forze che dirigono l' affacciamento del singoli nucleosomi in una flbra a struttura elicoidale e la configurazione permessa dalle costrizioni dei linkers.

\section{TRA PRESENTE E FUTURO}

I laboratori di fisica che si occupano specificatamente degli avanzamenti strumentali di questa tecnica fanno già Intravvedere alcuni sviluppi che permetteranno a questa microscopia di aprire ulteriori prospettive alla biologia strutturale. Fra questi è già in grossa evidenza la costruzione di microscopi integrati. L' abbinamento con un microscopio ad epifluorescenza invertito permette di osservare un campione con risoluzioni sia molecolari che macroscopiche, quali quelle della microscopia ottica.

Si sta sviluppando una vera ingegneria strutturale delle sonde che prevede anche la loro funzionalizzazione per mettere in gloco riconosclmenti molecolari fra molecole depositate sulla punta e molecole sul campione. Su questa linea

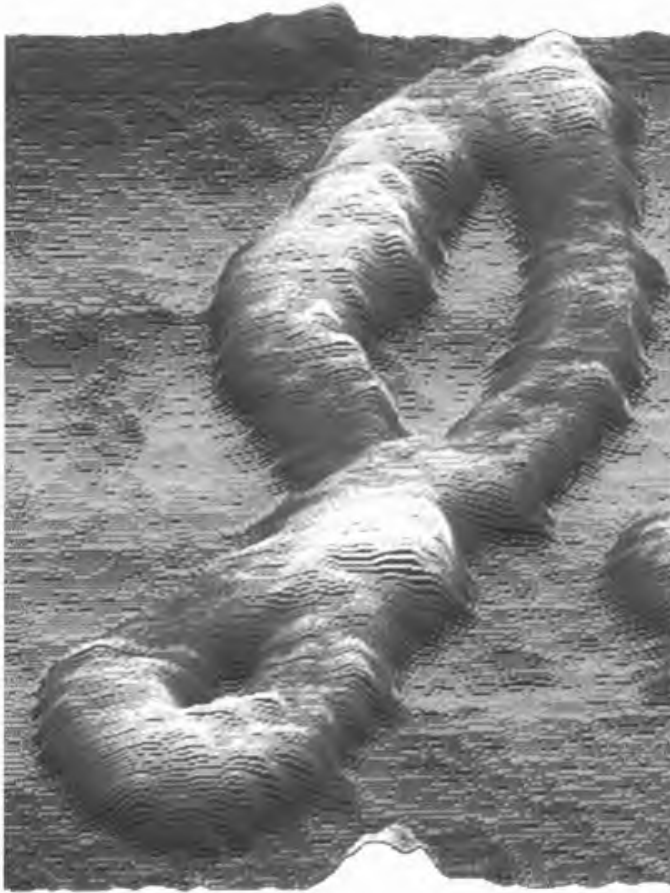

6. Immagine di una molecola di DNA ottenuta con la Microscopia a Forze in Scansione. E' stata questa la prima immagine tridimensionale del DNA in cui si è potuto riconoscere direttamente, oltre alla forma della molecola anche il superavvolgimento destrogiro della catena (da referenza 16)

si può pensare anche ad interazioni antigene-anticorpo per riconoscimenti localisu superfici cellulari.

$E^{\prime}$ in arrivo un microscopio noncontact, in cui la costruzione della topografia del campione non richiederà più alcun suo contatto meccanico con la sonda.

Si potranno sviluppare tecniche di dissezione molecolare. La punta dopo essere scorsa gentilmente su una molecola per permettere di costruirne l'immagine, può essere fermata su diversi punti di essa ed aumentando la pressione essere usata come un coltello per sezionare meccanicamente la molecola stessa Queste manipolazioni meccaniche di singole molecole permettono di ipotizzare anche una sorta di chirurgla su scala molecolare.

\section{CONCLUSIONI}

Questa tecnica nuovissima ancora ad uno stadio pionieristico $\mathrm{cl}$ sta rivelando glorno dopo glorno le sue sorprendenti potenziallta per la biologia strutturale. Ottenere con essa immagini a bassa 
risoluzione non è di norma difficile se il campione non è troppo "soffice", ma se si vogliono spingere i giochi al limite delle sue potenzialita i glochi diventano molto complessi. Gll svlluppi più recenti di questa tecnica evidenziano sempre piú chiaramente come essa debba operare in un contesto culturale e tecnologico fortemente interdisciplinare. Per lavorare al meglio nel laboratorio di biologia in cui questo strumento si viene a collocare devono essere disponibili sia microscopi ottici ed elettronici sia un laboratorio chimico per interventi di ingegnerizzazione delle sonde, in funzione del tipo di campione che si vuole studiare. Con questa microscopia non v'è una interazione di una radiazione con un campione come nelle altre microscopie classiche, ma interazioni meccaniche e chimico-flsiche fra due corpi: la sonda ed il campione. Le variabili in gioco sono ben più varie $\mathrm{e} \mathrm{di}$ ben più difficile controllo. Su questa base si capisce perchè a fianco di alcuni laboratori che possono esibire risultati sorprendenti ve ne siano molti altri che hanno grosse difficoltà nei giustlficare l' impegno fino ad ora profuso con questa tecnica e sono giunti al punto di meditare di abbandonarla

\section{BIBLIOGRAFIA}

1. C. Bustamante et al. Current Opinions Struct. Biol. 3 (1993) 363.

2. G. Binning, C. F. Quate \& C. Gerber, Phys, Rev. Lett. 56 (1980) 930

3. M. Bezanilla, B. Drake, E. Nudier, M. Kashlev, P.K. Hansma and H. Hansma: Motion and Enzymatic Degradation of DNA in the Atomic Force Microscope (in corso di stampa).

4. D. Sarld Scanning Force Microscopy: with applications to electric, magnetic and atomic forces Oxford University Press, 1991

5. J. Israelachvili Intermoiecular and surface forces Academic Press, 1985

6. S. Kasas, A. Berdal and M. R. Cello Scanning Probe Microscopies // (1993)

7. B. Drake et al. Science 243 (1989) 1586.

8 W. Haberle et al. Ultramicroscopy 42-44, (1992) 1161

9. S. Kasas et al. Biophys. J. 64 (1993) 539

10. A. L. Welsenhorn et al. Nanotechnology 4 (1993) 106.

11. S. Kasas, F. Broccoll and B. Samorl (in corso di pubbllcazione)

12. D. Keller e C. C. Chung Surface Science $\mathbf{6 8}$ (1992) 33.

13. W. A. Rees et al. Science 260 (1993) 1646.

14. A. J.J. Dietrich C. A. J. Putman, J. van Marle,
C. Heyting B. G. de Groth e J. Greve (Tn corso di pubbllcazione)

15. B. Samorì, G. Siligardi, C. Quagliariello, A. L Welsenhorn, J. Vesenka and C. J. Bustamante Proc. Nat. Acad. Sci. (USA) 90 (8) 1993, 3595-3601 .

16. B. Samorì, C. Nigro, V. Armentano, S. Cimieri, G. Zuccherì, C. Quagliariello Engl. Angew. Chem. Int Ed. (1993) 32, 1461-1463

17. G. Zuccheri, C. Nigro, G. Ranieri e B. Samori (in corso di pubblic.)

18. S. H. Leuba, G. Yang, B. Samorì, K. van Holde, J. Ziatanova, C. Bustamante (in corso di pubblicazione)

19. F. Thoms et al. J. Cell Biology 83 (1979) 403; Methods in Enzymology 170 (1989) 141.

20. Sperling e A. Tardieu FEBS Lett. 64 (1976) 89.

21. A.M. Campbell et al. Nucil. Acids Res. 15 (1978) 1571: L. Perez-Grau et al. Nucil. Acids Res. 12 (1984) 2987; S.E. Gerchman et al. Proc Natil. Acad. Sci. USA 84 (1987) 7802.

22. J. R. Swerdiow et al. Curr. Opin. Cell Biol. 84 (1993) 412.

* da un seminario tenuto il 15/2/94 presso il Dipartimento di Biologia Evol. Sper. dell' Università di Bologna .

* Dipartimento di Chimica, UNICAL Cosenza

\title{
Biodinâmica
}

\section{Biónica Aplicada Lda.}

\author{
RUA DA GUINÉ, $2-2^{\circ} \mathrm{E}$ \\ 1100 LISBOA-PORTUGAL \\ TEL. 8150760 - FAX 8150770
}

\section{INSTRUMENTAÇÃO}

HI-TECH SCIENTIFIC - Stopped Flow e instrumentaçāo para estudos de cinética de reacçōes rápidas.

PHOTON TECHNOLOGY INTERNATIONAL (PTI) - Fontes de Radiação, Fluorímetros (estado estacionário e de tempos de vida), Lasers de Azoto com ou sem laser de corantes, Fluorescência de Rácio, software.

IBH - Tempos de vida, Lâmpadas pulsadas, Detecção ultra rápida (fotomultiplicadores e instrumentação), software.

OLIS - Espectrofotómetros clássicos modernizados. Monocromadores de Scanning Rápido (até 1000 scans/sec).

CANBERRA INDUSTRIES - Instrumentação nuclear, detectores de estado sólido, etc.

BROOKHAVEN INSTRUMENTS - Analisadores de tamanho de partículas por dispersão de luz, centrifugação e electrocinética.

KINETIC SYSTEMS - Mesas e "breadboards" para óptica.

GENTEC - Medidores de energia para lasers.

LASER SHIELD - Óculos de protecção para radiação laser (Nd-Yag, $\mathrm{CO}_{2}, \mathrm{He}-\mathrm{Ne}$ ), espectro largo e UV.

CORION - Gama completa de filtros ópticos

STRAWBERRY TREE COMPUTERS - Placas e software para aquisição de dados.

HELLMA - Células (cuvettes) em vidro e quartzo.

Desenvolvimento e construção de instrumentação.

\section{Exponha-nos as suas necessidades}

